\title{
MODELO PREDITIVO DO POTENCIAL DE DISTRIBUIÇÃO GEOGRÁFICA DA ESPÉCIE Richeria grandis VAHL. NO ESTADO DE MINAS GERAIS E SUA RELAÇÃO COM AS FLUTUAÇÕES CLIMÁTICAS DO QUATERNÁRIO
}

Cristiane Coelho de Moura ${ }^{1}$, Thaís Ribeiro Costa ${ }^{1}$, Paula Alves Oliveira ${ }^{2}$, Marcelo Leandro Bueno ${ }^{3}$, Evandro Luiz Mendonça Machado ${ }^{4}$

${ }^{1}$ Mestranda do Programa de Pós-graduação em Ciência Florestal, Universidade Federal dos Vales do Jequitinhonha e Mucuri, Diamantina, Minas Gerais.

(Kinha_dtna@yahoo.com.br)

${ }^{2}$ Doutoranda do Programa de Pós-graduação em Ciência Florestal, Universidade

Federal dos Vales do Jequitinhonha e Mucuri, Diamantina, Minas Gerais.

${ }^{3}$ Residente de Pós-doutorado na Universidade Federal de Viçosa - Laboratório de Ecologia e Evolução de Plantas.

${ }^{4}$ Professor adjunto do curso de Engenharia Florestal e Coordenador do programa de Pós-graduação em Ciência Florestal, Universidade Federal dos Vales do Jequitinhonha e Mucuri.

\section{Recebido em: 08/04/2017 - Aprovado em: 10/06/2017 - Publicado em: 20/06/2017 DOI: 10.18677/EnciBio_2017A73}

\begin{abstract}
RESUMO
O objetivo deste trabalho foi avaliar o efeito das flutuações climáticas do Quaternário sobre a distribuição preditiva da espécie Richeria grandis VAHL. no estado de Minas Gerais. Foram utilizados dados de ocorrência da espécie e 10 variáveis climáticas referentes a três cenários passados (Holoceno, Último Glacial Máximo e Interglacial) e período atual para a análise da modelagem. O algoritmo Maxent foi selecionado para relacionar a ocorrência da espécie com variáveis bioclimáticas que refletem diferentes condições de temperatura, precipitação e sazonalidade. Os modelos foram validados por meio do índice Area Under Curve (AUC) e a inflluência das variáveis sobre a distribuição foi avaliada por meio do teste Jackknife. Os modelos apresentaram bom desempenho, visto que os índices AUC variaram entre 0,81 a 0,87 . Os modelos do Quaternário evidenciaram um aumento gradativo da adequabilidade para a Richeria grandis nos cenários do Interglacial (120 - 140 mil anos atrás), Último Glacial Máximo (22 mil anos atrás) e Holoceno (6000 anos atrás), respectivamente. Já para o cenário atual (1960 - 1990), houve uma baixa de adequabilidade, quando comparado ao período Holoceno. As variáveis bioclimáticas que melhor contribuíram para a modelagem foram: Precipitação do mês mais chuvoso, a qual parece ter a maior informação útil agregada, seguida da variável Precipitação do mês mais seco, Isotermalidade e por último a Sazonalidade de temperatura. Esta ferramenta mostrou-se bastante útil em fornecer contribuições para o estudo de questões ecológicas sobre a distribuição da espécie, além de ser uma importante ferramenta para planos de conservação da biodiversidade.
\end{abstract}

PALAVRAS-CHAVE: mata de galeria, maxent, modelagem. 


\title{
PREDICTIVE MODEL OF THE POTENTIAL OF GEOGRAPHICAL DISTRIBUTION OF THE SPECIES Richeria grandis VAHL. IN THE STATE OF MINAS GERAIS AND ITS RELATIONSHIP AS CLIMATIC FLOATING OF THE QUATERNÁRIO
}

\begin{abstract}
The objective of this work was to evaluate the effect of Quaternary climate fluctuations on the predictive distribution of the Richeria grandis VAHL. species in the state of Minas Gerais. We used data of the occurrence of the species, and 10 climatic variables referring to three past scenarios (Holocene, Last Glacial Maximum and Interglacial) and current period for modeling analysis. The Maxent algorithm was selected to relate the occurrence of the species with bioclimatic variables that reflect different temperature, precipitation and seasonality conditions. The models were validated using the AUC index and the influence of the variables on the distribution was evaluated through the Jackknife test. The models presented good performance, since the AUC indexes ranged from 0.81 to 0.87 . The Quaternary models showed a gradual increase in suitability for Richeria grandis in the Interglacial (120 - 140 thousand years ago), Last Glacial Maximum (22 thousand years ago) and Holocene (6000 years ago) scenarios respectively. For the current scenario (1960-1990), there was a decrease in adequacy when compared to the Holocene period. The bioclimatic variables that best contributed to the modeling were: Precipitation of the wettest month, which seems to have the greatest useful information aggregated, followed by the variable Precipitation of the driest month, isothermality and finally the Seasonality of temperature. This tool has proved to be very useful in providing contributions to the study of ecological issues on the species distribution, as well as being an important tool for biodiversity conservation plans.
\end{abstract}

KEYWORDS: gallery forest, maxent, modeling.

\section{INTRODUÇÃO}

Os padrões de distribuição e o intercâmbio de espécies entre diferentes ecossistemas são dependentes de configurações geográficas e eventos históricos em escalas regionais (MEDEIROS, 2009). Fatores intrínsecos dos organismos, a heterogeneidade ambiental e a pressão antrópica, constituem alguns dos importantes mecanismos reguladores da riqueza e abundância de espécies nas comunidades.

Estudos revelam que o domínio Cerrado, no passado, era responsável pela conexão entre a biota dos demais biomas, como a Amazônia e a Mata Atlântica, corroborado pelos padrões de distribuição disjunta em várias espécies da flora desses ambientes (RATTER et al., 1973). Os efeitos das flutuações climáticas sobre a vegetação do Cerrado durante o período Quaternário (WERNECK et al., 2012; BUENO et al. 2017) confirmam que as formações florestais e as áreas de cerrado tiveram sucessivas expansões e retrações, promovendo áreas de conexão entre os domínios fitogeográficos. OLIVEIRA-FILHO \& RATTER (2002) sugeriram que a rede formada por matas de galeria do Brasil Central funciona como corredores ecológicos, atuais e pretéritos interligando a floresta amazônica à floresta atlântica, no sentido noroeste-sudeste, representando um vetor de grande relevância para a compreensão dos padrões de distribuição das espécies. Neste contexto, as matas de galeria destacam-se por apresentar importante papel na proteção dos recursos naturais e conservação da biodiversidade (OLIVEIRA et al., 2015; SILVA et al., 2016). 
Apesar das florestas de galeria estarem amparadas legalmente pela Lei ( $n^{\circ}$ 12.651 de 25/05/2012; alterada pela Lei №. 12.727 de 17/10/2012), a degradação acelerada tem promovido a descontinuidade desses ambientes em ilhas isoladas. A fragmentação dos ecossistemas pode comprometer o fluxo e a dispersão de espécies (MAUÉS et al., 2010) e promover alterações na diversidade genética das populações naturais (MOREIRA, 2012), sujeitando diversas espécies, em especial as exclusivas desses ambientes, ao processo de declínio populacional e extinções locais (LARANJEIRA, 2012). Quanto menores os espaços ecológicos nesses fragmentos, mais urgentes se torna o estabelecimento de modelos de conservação de sua biodiversidade, que possam orientar o delineamento de políticas públicas de restauração e manejo racional de seus recursos.

Um método que vem sendo amplamente usado para a definição de áreas prioritárias para a conservação é a modelagem de nicho ecológico (WERNECK et al. 2012; BUNGER et al. 2016; BUENO et al. 2017). Este método permite predizer a distribuição potencial de espécies, com base em áreas de ocorrência conhecidas, variáveis ambientais e em requerimentos ecológicos das mesmas, ou seja, características que compõem o nicho (PHILIPS et al., 2006; LIMA-RIBEIRO \& DINIZ FILHO, 2012).

Diante disso, o objetivo do presente estudo foi avaliar o efeito das flutuações climáticas do Quaternário sobre a distribuição preditiva da espécie Richeria grandis Vahl. no estado de Minas Gerais.

\section{Área de Estudo}

\section{MATERIAL E MÉTODOS}

A área de estudo é representada pelo estado de Minas Gerais (Figura 1), em particular nas mesorregiões do Triângulo Mineiro, região metropolitana de Belo Horizonte (BH), Jequitinhonha, Central Mineira e Norte de Minas. O estado foi selecionado por ser o que apresenta o maior número de registros da espécie no Brasil. De maneira geral, o estado apresenta 4 tipos climáticos pela classificação de Koppen, sendo eles Cwb, Aw e BSw (EMBRAPA, 2017).

Nas regiões que compreende a Serra do Espinhaço, com altitudes mais elevadas, o clima é subtropical (Cwb), com inverno seco e verão ameno (EMBRAPA, 2017). O clima predominante no Norte de Minas Gerais e em uma pequena área do Vale do Jequitinhonha é o BSw, caracterizado por um clima seco com chuvas no verão, com precipitações anuais sempre inferiores a $1000 \mathrm{~mm}$ e normalmente inferiores a $750 \mathrm{~mm}$ (EMBRAPA, 2017).

O clima que predomina na maior parte das regiões de Minas Gerais amostradas neste estudo é o Aw. Esta classificação é caracterizada pelo clima tropical, com inverno seco, apresentando estação chuvosa no verão e nítida estação seca no inverno. A temperatura média do mês mais frio é superior a $18^{\circ} \mathrm{C}$ e as precipitações são superiores a $750 \mathrm{~mm}$ anuais, atingindo $1800 \mathrm{~mm}$ (EMBRAPA, 2017). 


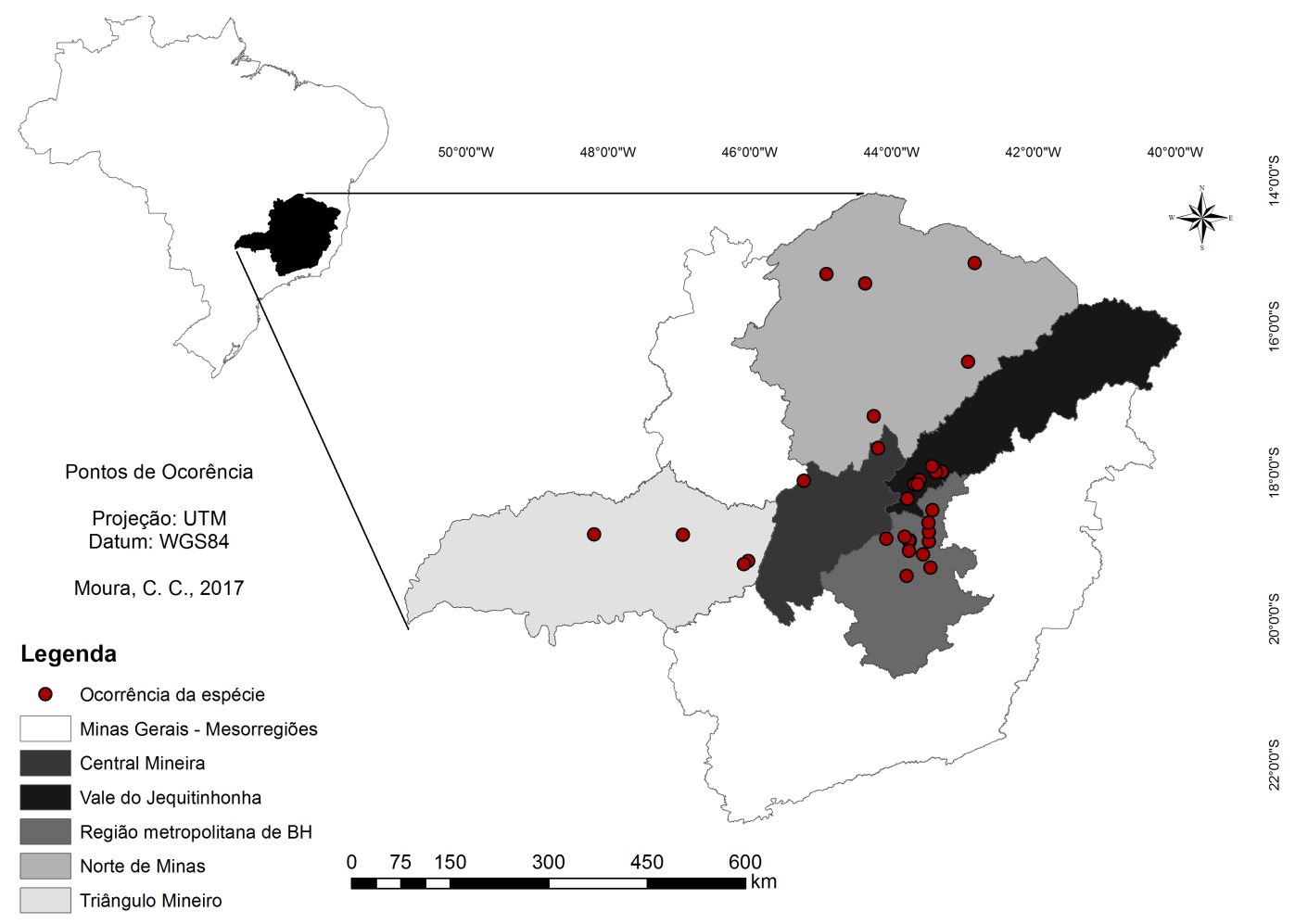

FIGURA 1. Pontos de coleta da espécie Richeria grandis VAHL. inseridos nas mesorregiões, Minas Gerais.

Foram utilizados dados de ocorrência da Richeria grandis (107 pontos) registrados em vários herbários brasileiros (informações sobre a biodiversidade brasileira (GIANNINI et al., 2012) e disponíveis no site do SPECIESLINK (2017). Durante o processamento dos dados, pontos de ocorrência duvidosos, com localidade incompleta ou incerta, e com coordenadas repetidas foram excluídos da pesquisa.

\section{Espécie de estudo}

A espécie em estudo, Richeria grandis VAHL (Phyllanthaceae) (IPNI, 2012), é uma espécie indicadora de solos saturados por água e pode ser destacada como importante espécie de mata de galeria inundável (SILVA, 2012; MARTINS, 2015; SILVA et al. 2016). Essa espécie distribui-se ainda da Amazônia até a Floresta Atlântica, atravessando o Brasil Central pelas matas de galeria, alcançando as florestas de brejo, em meio à vegetação de Caatinga (RIBEIRO-FILHO et al., 2009). Porém, o estado com maior número de registros da espécie no Brasil é Minas Gerais (FLORA DO BRASIL, 2017).

\section{Seleção das Variáveis, construção do modelo e validação do modelo}

Foram utilizadas 19 variáveis bioclimáticas com resolução de aproximadamente $1 \mathrm{Km}$ (30 arc-segundos). As variáveis bioclimáticas são derivadas dos valores mensais de temperatura e precipitação utilizadas na modelagem de distribuição de espécies, e representam tendências anuais, sazonalidade e fatores ambientais extremos ou limitantes (HIJMANS et al., 2005). As camadas foram cortadas seguindo a área de estudo, ou seja, o estado de Minas Gerais.

Afim de produzir modelos para inferir a paleodistribuição de Richeria grandis, foram produzidas projeções de adequabilidade de ocorrência para o presente (19601990), Médio-Holoceno (6ka antes do presente - AP), Último Glacial Máximo (LGM - 
21ka AP) e Último Interglacial Máximo (LIG - 120ka AP), períodos baseados nas simulações climáticas (<www.worldclim.org>; HIJMANS et al., 2005). Para os períodos UGM, Holoceno e presente, empregou-se o Modelo de Sistema Climático Comunitário - CCSM4 (GENT et al., 2011), que representa dados de simulações climáticas com modelos climáticos globais (GCMs) baseado no Projeto de Intercomparação do Modelo Acoplado Fase 5 (CMIP5, TAYLOR et al., 2012). O modelo paleoclimático para O último Interglacial (120 ka, LIG) utilizou a abordagem de OTTO-BLIESNER et al. (2006). Foi utilizado o ArcGIS ver. 10.1 (ESRI, 2011) para todo o sistema de informação geográfica (SIG).

Com o objetivo de selecionar as variáveis bioclimáticas mais representativas para o estudo e evitar presença de multicolinearidade entre as variáveis, realizou-se o teste de correlação de Pearson. Aquelas altamente correlacionadas $(r>0,9$ ou $<$ - 0,9) foram excluídas da modelagem com base na relevância ecológica, reduzindo a chance de ocorrer o sobreajustamento dos modelos. Para a verificar a modelagem de nico ecológico de Richeria grandis, foi utilizado o programa Maximun Entropy Species Distribution Modelling (MaxEnt) na versão 3.3.3k (PHILLIPS et al., 2006).

O Maxent é um método capaz de realizar previsões ou inferências de informações incompletas, oferecendo diversas vantagens comparado a outros métodos (ELITH \& GRAHAM, 2009). Dentre as vantagens, destaca-se o fato de necessitar apenas de dados de presença para modelar toda a área de estudo, juntamente com as informações ambientais (PHILIPS et al., 2006). O Maxent é amplamente utilizado em trabalhos de modelagem de nicho ecológico (MDE), tendo mostrado melhor resultado de predição em estudos que comparam diferentes MDE (ELITH \& GRAHAM, 2009; GIOVANELLI et al., 2010; TARKESH \& JETSCHKE, 2012) além de resultados satisfatórios com poucos pontos de presença (WILLIAMS et al., 2009).

Uma vez que esse algoritmo também fornece uma curva de resposta da contribuição de cada variável, utilizou-se o teste de Jackniffe de acordo com PHILLIPS et al. (2006), para estimar quais foram as variáveis bioclimáticas de maior contribuição no modelo.

No software Maxent foram utilizadas 5000 interações com 10 repetições do tipo subsample, para se obter uma superfície de possibilidade de distribuição mais robusta. Para avaliar as predições realizadas, foi utilizada a área sobre a curva ROC (AUC - Area Under Curve). O índice AUC é referente à probabilidade ao acaso da identificação correta dos locais sem informação de presença (PHILLIPS \& DUDIK, 2008). Um teste de validação pode adotar os valores de AUC a seguir como indicadores da qualidade do modelo (METZ, 1986): excelente $(1,0 \square 0,9)$; bom $(0,9$ $\square 0,8)$; médio $(0,8 \square 0,7)$; ruim $(0,7 \square 0,6)$; muito ruim $(0,6 \square 0,5)$.

A AUC serve como uma medida de avaliação do modelo independente de um limite de corte escolhido (PHILLIPS \& DUDIK, 2008). Esta é um indicador direto da capacidade discriminatória do modelo e é interpretada diretamente como a probabilidade do modelo em que, dado um ponto de verdadeira presença e um ponto de verdadeira ausência, o modelo será classificado corretamente (PHILLIPS et al., 2006).

Tanto o cálculo do índice AUC, quanto o teste estatístico Jackknife foram calculados pelas ferramentas de pós-análise disponíveis no software Maxent. Para inferir sobre o efeito das mudanças climáticas sobre a espécie no estado de Minas Gerais, foram comparadas as projeções nos cenários passado e presente. Para cada pixel, o Maxent indica um valor numérico que varia de 0-1, melhor definido 
como adequabilidade ambiental e não como probabilidade de ocorrência (PEARSON et al., 2007), sendo representado nos mapas por meio de uma escala de cor crescente, onde os tons mais escuros constituem uma maior adequabilidade ambiental da espécie em estudo.

Foi realizado um mapa contendo dados geoespaciais em forma de vetor da área do domínio Cerrado presente no território de Minas Gerais, bem como os cursos d'água e principais rios, a fim de comparação das projeções modeladas com outros fatores ambientais, no cenário atual. Os shapefiles foram obtidos do site do IEDE (2017). Todas as etapas pré e pós modelagem foram realizadas utilizando o ArcGIS ver. 10.1 (ESRI 2011) para todo o sistema de informação geográfica (SIG).

\section{RESULTADOS E DISCUSSÃO}

A modelagem de distribuição para o período atual apresentou um índice de AUC de 0,871 e desvio padrão de $\pm 0,066$. Para os modelos de distribuição dos cenários passados, Holoceno, Médio Glacial Máximo e Interglacial, os valores de AUC foram de 0,855, 0,816 e 0,859, com desvio padrão de $\pm 0,0,037, \pm 0,038$, e \pm 0,046 , respectivamente. As variáveis bioclimáticas correlacionadas mantidas para a modelagem foram: bio 1 (Temperatura média anual), bio 2 (Variação diurna média de temperatura), bio 3 (Isotermalidade), bio 4 (Sazonalidade de temperatura), bio 6 (Temperatura mínima do mês mais frio), bio 7 (Amplitude térmica atual), bio 12 (Precipitação anual), bio 13 (Precipitação do mês mais chuvoso), bio 14 (Precipitação do mês mais seco) e bio 18 (Precipitação do trimestre mais quente).

Os modelos do período Quaternário, evidenciaram um aumento gradativo da adequabilidade para a Richeria grandis, nos cenários do Interglacial (120 ka AP), Último Glacial Máximo (21 ka AP) e Holoceno (6 ka AP), respectivamente (Figura 2).

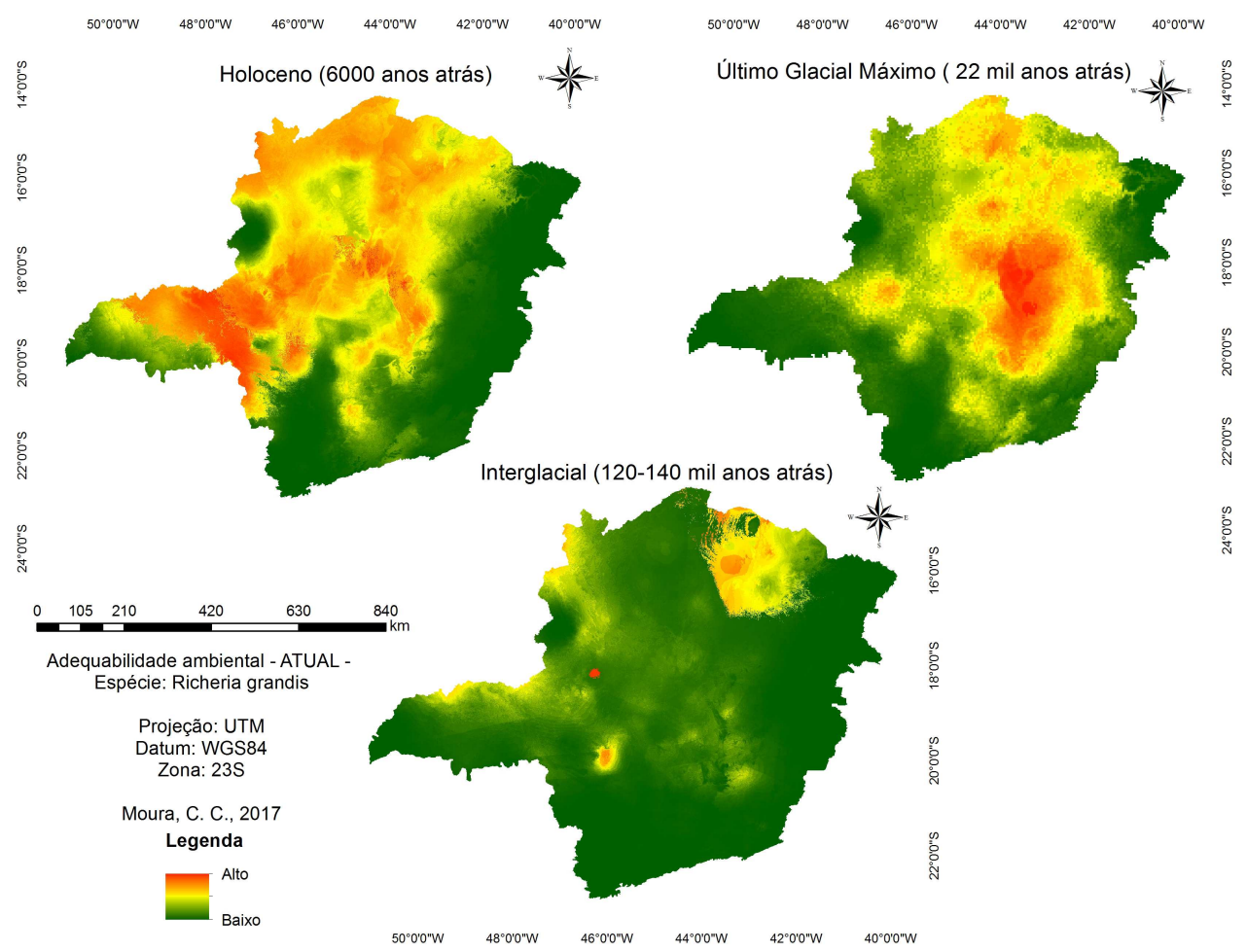

FIGURA 2. Mapas de distribuição potencial do cenário Holoceno (6ka AP), Último Glacial Máximo (21ka AP) e Interglacial (120ka AP) da espécie Richeria grandis no estado de Minas Gerais, com percentual de adequabilidade ambiental da espécie. 
Já para o cenário atual (1960 - 1990), houve uma baixa de adequabilidade, quando comparado ao período Holoceno. A espécie $R$. grandis, apresentou-se com uma maior distribuição no domínio Cerrado e transição com a Mata Atlântica, onde há uma maior ocorrência dos cursos d’água e os rios principais (Figura 3).

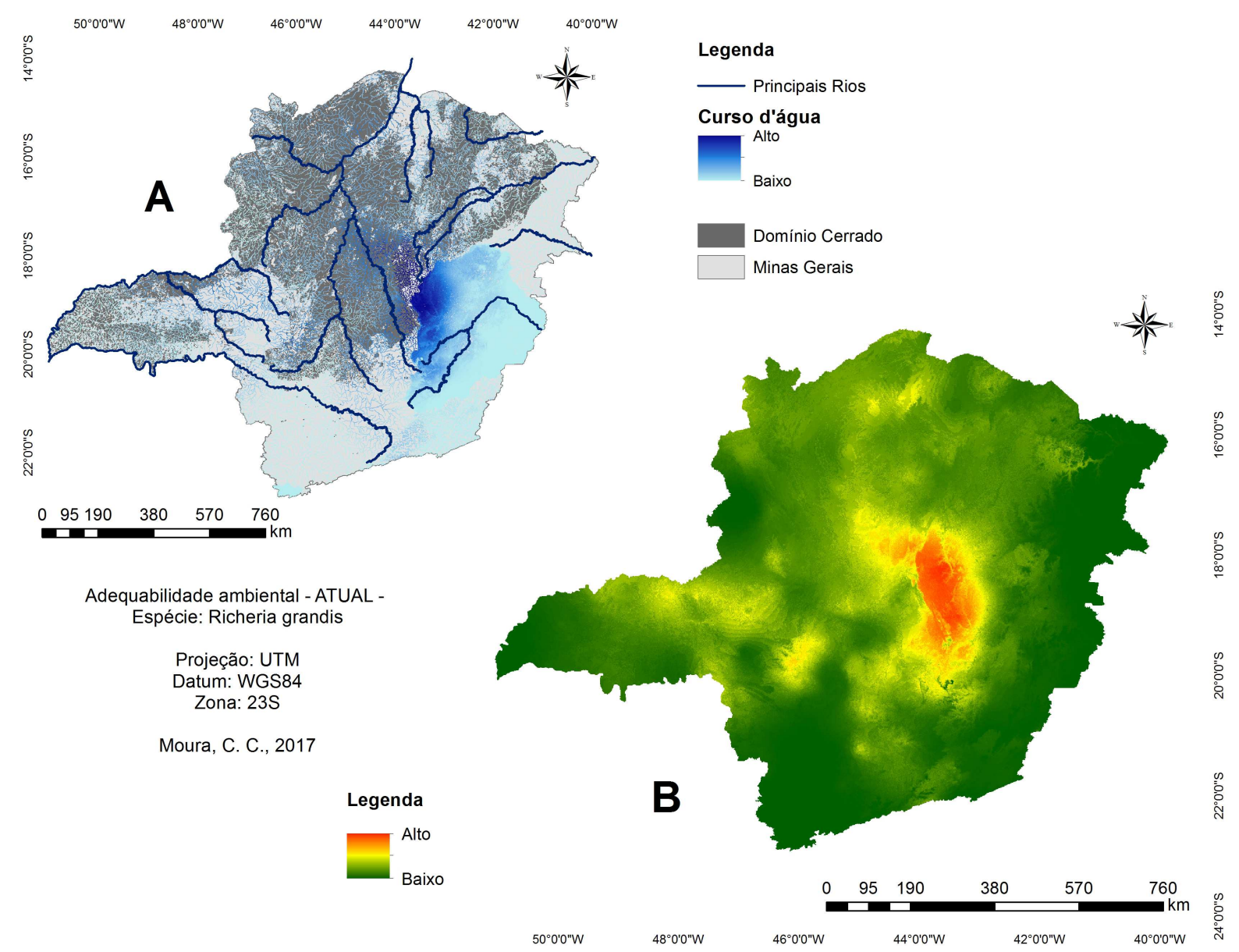

FIGURA 3. Mapa de Minas Gerais contendo a localização da área do domínio Cerrado, juntamente com os cursos d'água e principais rios (A). Mapa de distribuição potencial do cenário atual (1960-1990) da espécie Richeria grandis no estado de Minas Gerais, com percentual de adequabilidade ambiental da espécie.

As variáveis bioclimáticas que melhor contribuíram para a modelagem das espécies e que favorece a ocorrência da $R$. grandis, de acordo com o resultado de Jackknife foram as: bio 3 , bio 4 , bio 13 e bio 14 para todos os cenários passados, e também para o atual (Tabela 2). Destas, verifica-se que a variável ambiental com ganho mais elevado, e maior perda quando se omite, quando usada isoladamente é a precipitação do mês mais chuvoso (bio 13), a qual parece ter a maior informação útil agregada, seguida da variável precipitação do mês mais seco (bio 14), Isotermalidade (bio 3) e por último a Sazonalidade de temperatura (bio 4). 
TABELA 2. Valores percentuais de contribuição relativa das principais variáveis ambientais para cada modelo de adequabilidade ambiental da Richeria grandis em Minas Gerais

\begin{tabular}{lcccc}
\hline & \multicolumn{4}{c}{ Porcentagem de contribuição (\%) } \\
& Bio 3 & Bio 4 & Bio 13 & Bio 14 \\
\hline Interglacial & 18,9 & 6,7 & 45,3 & 26,7 \\
Último Glacial Máximo & 19,8 & 9 & 43,5 & 25,5 \\
Holoceno & 16,8 & 9,2 & 46,8 & 21,9 \\
Atual & 18,1 & 9,3 & 42,4 & 26,9 \\
\hline
\end{tabular}

Os resultados para o AUC apresentaram valores significantes para a qualidade dos modelos. Neste sentindo, segundo METZ (1986), todas as curvas apresentaram uma validação de boa qualidade, uma vez que todas se encontram no intervalo de $0,8-0,9$.

É notório que a $R$. grandis apresentou uma adequabilidade ambiental condizente com o observado por meio dos pontos de coleta (Figura 1), uma vez que os mapas gerados pela modelagem (Figura 2 e 3 ) apresentam maior intensidade de adequação na mesma região onde se localiza os dados de ocorrência da espécie, exceto para o período interglacial.

As variações nos ciclos orbitais da Terra (Teoria de Milankovitch) alteram a quantidade de energia solar recebida pelo planeta, ocasionando assim, em alterações climáticas (MARTINS, 2016). Os períodos Interglacial (LIG) e Último Glacial Máximo (LGM), marcados por mudanças nos padrões de circulação oceânica e atmosférica (MOLBESCH et al., 2012), são caracterizados por apresentarem padrões climáticos significadamente mais frios e secos (BARBOSA \& FERNANDES, 2016). Apesar de apresentar condições secas e frias, o LGM não foi um período de extrema aridez (MOSBLECH et al., 2012), vindo a justificar a maior adequabilidade da $R$. grandis neste período quando comparado ao LIG. Enquanto que para o Holoceno, a maior área de adequabilidade ambiental se justifica pelo padrão de clima mais quente e úmido no período (BARBOSA \& FERNANDES, 2016).

Neste sentido, os períodos em que se caracteriza como quentes e úmidos, as florestas se expandiram, e nos períodos mais frios e secos houve retração das florestas dando lugar para áreas de campo (CONCEIÇÃO et al., 2016) o que corrobora com o resultado deste estudo, uma vez que, quando ocorreram eventos de retração das florestas (LIG e LGM), a $R$. grandis, que é uma espécie arbórea associada a remanescentes de florestas, não teve muita adequabilidade ambiental, se comparado ao Holoceno (quente e úmido).

Contudo, pode-se atrelar esta baixa adequabilidade ambiental atual, às grandes pressões antrópicas sobre as matas de galeria, típicas do Cerrado (MARCON, 2013; RIBEIRO, 2013; MARTINS, 2014; JESUS, 2016) acarretando mudanças no microclima local, e transformando-se em áreas que a $R$. grandis não consiga sua adaptabilidade, uma vez que ela é indicadora de solos saturados por água e pode ser destacada como importante espécie destas matas (SILVA \& WALTER, 2011; SILVA, 2012; MARTINS, 2015; SILVA et al. 2016), onde segundo MARÇAL (2016) as influências climáticas podem definir as condições de limites do fluxo dentro dos quais os rios operam (MARÇAL, 2016).

Além do mais, as variáveis que mais contribuem para a adequabilidade da $R$. grandis são aquelas relacionadas à precipitação, chegando a um percentual somado 
de $69,3 \%$ (Tabela 2) para o período atual, confirmando o indicativo da $R$. grandis estar presentes em solos saturados por água e, a perda deste fator possa vir a excluí-la destes ambientes adequados para sua existência.

\section{CONCLUSÃO}

Richeria grandis apresentou uma variação para a adequabilidade ambiental influenciada pelas flutuações climáticas no período Quaternário e atual. As variáveis climáticas que mais contribuíram para uma boa modelagem são as que se referem à precipitação. A modelagem preditiva apresentou maior intensidade de adequação na mesma região onde se localizam os dados de ocorrência da espécie.

\section{REFERÊNCIAS}

BARBOSA, N. P. U.; FERNANDES, G. W. Rupestrian grassland: past, present and future distribution. In: Ecology and Conservation of Mountaintop Grasslands in Brazil. Fernandes, G. W. (Ed.), 2016.

BRASIL. Lei n. 12.651, de 25 de Maio de 2012. Dispõe sobre a proteção da vegetação nativa e dá outras providências. Disponível em: <http://www.planalto.gov.br/ccivil_03/_ato2011-2014/2012/lei/l12651.htm>, acesso em: 17 de Fevereiro de 2017.

BUENO, M. L.; PONTANA, V.; NEVES, D. M.; RATTER, J. A.; OLIVEIRA-FILHO, A. T. Effects of Quaternary climatic fluctuations on the distribution of Neotropical savanna tree species. Ecography, v. 40, n. 403-414, 2017. Doi:10.1111/ecog.01860.

BUNGER, M. O.; MAZINE, F. F.; FOREST, F.; BUENO, M. L.; STEHMANN, J. R.; LUCAS, E. J. The evolutionary history of Eugenia sect. Phyllocalyx (Myrtaceae) corroborates historically stable areas in the southern Atlantic forests. Annals of Botany, v. 118, n. 1209-1223, 2016. Doi: 10.1093/aob/mcw209, Disponível em: www.aob.oxfordjournals.org..

CONCEIÇÃO, A. A.; RAPINI, A.; CARMO, F. F.; BRITO, J. C.; SILVA, G. A.; NEVES, S. P. S.; JACOBI, C. M. Rupestrian grassland vegetations, diversity, and origin. In: FERNANDES, G. W. Ecology and Conservation of Mountaintop grasslands in Brazil, 2016.

ELITH, J.; GRAHAM, C. H. Do they? How do they? WHY do they differ? On finding reasons for differing performances of species distribution models. Ecography, v. 32, n. $1, \quad$ p. 66-77, 2009. Disponível em: < http://onlinelibrary.wiley.com/doi/10.1111/j.1600-0587.2008.05505.x/full>. $\quad$ DOI: 10.1111/j.1600-0587.2008.05505.x

EMBRAPA FLORESTAS. Disponível em: http://www.cnpf.embrapa.br/pesquisa/efb/clima.htm>, acesso em Maio de 2017. GIANNINI, T. C.; SIQUEIRA, M. F.; ACOSTA, A. L.; BARRETO, F. C. C.; SARAIVA, A. M.; ALVES-DOS-SANTOS, I. Desafios atuais da modelagem preditiva de distribuição de espécies. Rodriguésia, v. 63, n.3, p. 733-749. 2012. Disponível em: $<\quad$ http://www.producao.usp.br/handle/BDPI/40715>. DOI: 10.1590/S217578602012000300017. 
GIOVANELLI, J. G. R.; de SIQUEIRA, M. F.; HADDAD, C. F. B.; ALEXANDRINO, J. Modeling a spatially restricted distribution in the Neotropics: How the size of calibration area affects the performance of five presence-only methods. Ecological Modelling, v. 221, n. 2, p. 215-224, 2010. Disponível em: < http://www.sciencedirect.com/science/article/pii/S0304380009006620> DOI: http://dx.doi.org/10.1016/j.ecolmodel.2009.10.009.

HIJMANS R. J.; GUARINO, L.; BUSSINK, C.; MATHUR, P.; CRUZ, M.; BARRENTES, I.; ROJAS, E. A geographic information system for the analysis of species distribution data. DIVA-GIS 7.5, 2012. Disponível em: <http://www.divagis.or>.

IEDE - Infraestrutura Estadual de Dados Espaciais de Minas Gerais. 2017. Disponível em <http://www.iga.mg.gov.br/IEDE>. Acesso em Janiero de 2017.

JESUS, E. O. Apropriação do Cerrado: análise do currículo e práticas educativas na rede estadual de educação de Goiás. CaderNAU- Cadernos do Núcleo de Análises Urbanas, v.9, n. 1, p.14. 2016. Disponível em: < https://seer.furg.br/cnau/article/view/6581>.

LARANJEIRA, M. Estrutura Espacial e Processos Ecológicos: O estudo da Fragmentação dos Habitats. Revista de Geografia e Ordenamento do Território, Centro de Estudos de Geografia e Ordenamento do Território. v.1, n. 1, p. 59-83. 2012.

LIMA-RIBEIRO, M. S.; DINIZ FILHO, J. A. F. Modelando a distribuição geográfica das espécies no passado: uma abordagem promissora em paleoecologia. Revista Brasileira de Paleontologia, v. 15, n. 3, p. 371-385. 2012.

MARÇAL, M. S.; LIMA, R. N. S. Abordagens conceituais contemporâneas na Geomorfologia Fluvial. Espaço aberto, PPGG- UFRJ, v. 6, n. 1, p. 17-33, 2016. Disponível em: < https://revistas.ufrj.br/index.php/EspacoAberto/article/view/5236>.

MARCON, J. B.; PESSOA, M. B.; PAVEI, P. T. Proposta de recuperação ambiental de uma área degradada pela ocupação urbana desordenada: estudo de caso bairro Cristo Redentor - Criciúma/SC. Cataventos: Revista de Extensão Universitária de Cruz Alta, v. 01, n. 05, p.131-145, dez. 2013. Disponível em: http://revistaeletronica.unicruz.edu.br/.

MARTINS, G. S. Reconstrução paleoclimática e paleohidrológica em registros sedimentares de lagos amazônicos. Niterói, 2016. 186 p. Tese (doutorado em Geociências e Geoquímica Ambiental) Universidade Federal Fluminense, 2016. Disponível em: < http://repositorio.uff.br/jspui/handle/1/2188>.

MARTINS, M.S.; Dinâmica do componente arbóreo em um trecho de mata de galeria inundável na Fazenda Sucupira, Brasília - DF, no período de 15 anos. 2015. viii, 63 f., il. Monografia (Bacharelado em Engenharia Florestal) Universidade de Brasília, Brasília, 2015. Disponível em: < http://bdm.unb.br/handle/10483/14949>. 
MARTINS, S. V. Recuperação de matas ciliares: no contexto do novo Código Florestal. 3ed - Viçosa, MG: Aprenda fácil, 2014. 220p.

MAUÉS, M. M.; OLIVEIRA, P. E. A. M. Consequências da fragmentação do habitat na ecologia reprodutiva de espécies arbóreas em florestas tropicais, com ênfase na Amazônia. Oecologia Australis, v. 14, n. 1, p. 238-250, 2010. Disponível em: < https://www.researchgate.net/profile/marcia_maues/publication/235989744_consequ encias_da_fragmentacao_do_habitat_na_ecologia_reprodutiva_de_especies_arbore as_em_florestas_tropicais_com_enfase_na_amazonia/links/0c960515441285978a0 00000. pdf >. DOI: $10.4257 /$ oeco. $2010.1401 . \overline{14}$.

MEDEIROS, D. P. W. Restingas: Aspectos fisionômicos e atributos biológicos em ecossistema adjacente à Floresta Atlântica do Centro de Endemismo Pernambuco. Tese de Doutorado em Botânica, UFRP, Recife. 127 p. 2009.

METZ, C.E. ROC methodology in radiologic imaging. Investigational Radiology, v. 21 , n. $9, \quad$ p. $720-733, \quad 1986$. Disponível em: < http://journals.Iww.com/investigativeradiology/Abstract/1986/09000/ROC_Methodolog y_in_Radiologic_Imaging.9.aspx>.

MOSBLECH, N. A. S. ; North Atlantic forcing of Amazonian precipitation during the last ice age. Nature Geoscience,v.5, p.817-820, 2012.

MOREIRA, P. A. Diversidade genética e efeitos da fragmentação de Matas Secas em Enterolobium contortisiliquum (Fabaceae): implicações para conservação. Tese de doutorado em Genética, UFMG, Belo Horizonte, 123 p. 2012. Disponível em: < http://www.bibliotecadigital.ufmg.br/dspace/handle/1843/BUOS8YVN96>.

OLIVEIRA FILHO, A.T.; RATTER, J.A. Vegetation physiognomies and woody flora of the cerrado biome. In The cerrados of Brazil. Ecology an natural history of a Neotropical savanna (P.S. Oliveira \& R.J. Marquis, eds.). Columbia University Press, New York, p.91-120, 2002.

OLIVEIRA, L. C. S.; GUIMARÃES, J. C. O.; SOUZA, I. C. S.; LIMA, C. M.; FERREIRA, W. C. Levantamento florístico e fitossociológico da regeneração natural de uma mata de galeria localizada no município de Jataí - GO. Global Science and Technology. V.08, n.03, p.59, 2015. Disponível em: < http://rv.ifgoiano.edu.br/periodicos/index.php/gst/article/view/764/483>. ISSN: 19843801.

PEARSON, R. G.; RAXWORTHY, C. J.; NAKAMURA, M.; TOWSEND, A. P. Predicting species distributions from small numbers of occurrence records: a test case using cryptic geckos in Madagascar. Journal of Biogeography, v. 34, n. 1, p. 102-117, 2007. Disponível em: < http://onlinelibrary.wiley.com/doi/10.1111/j.13652699.2006.01594.x/full>. DOI: 10.1111/j.1365-2699.2006.01594.x. 
PHILLIPS, S. J.; ANDERSON, R. P.; SCHAPIRE, R. E. Maximum entropy modeling of species geographic distributions. Ecological Modelling, v. 190, n. 3-4, p. 231259, 2006. Disponível em: < http://dx.doi.org/10.1016/j.ecolmodel.2005.03.026>.

PHILLIPS, S. J.; DUDIK, M. Modeling of species distributions with Maxent: new extensions and a comprehensive evaluation. Ecography, v. 31, n.2, p.161-175, 2008. Disponível em: < http://onlinelibrary.wiley.com/doi/10.1111/j.09067590.2008.5203.x/full>. DOI: 10.1111/j.0906-7590.2008.5203.x.

PRATI, R. C.; BATISTA, GEAPA; MONARD, M. C. Curvas ROC para avaliação de classificadores. Revista IEEE América Latina, v. 6, n. 2, p. 215-222, 2008. Disponível em:

http://www.ime.unicamp.br/ wanderson/Aulas/Aula11/artigo_curva_ROC.pdf>.

RATTER, J.A., RICHARD, P.W., ARGENT, G.T.; GIFFORD, D. R. Observations on the Vegetation of Northeastern Mato Grosso: I. The Woody Vegetation Types of the Xavantina-Cachimbo Expedition Area. Philosophical Transactions of the Royal Sociaty B - Biological Sciences. v. 266, n.880, n. 44-492, 1973. Disponível em: < http://rstb.royalsocietypublishing.org/content/266/880/449.short>.

DOI: 10.1098/rstb.1973.0053.

RIBEIRO - FILHO, A. A. FUNCH, L. S., RODAL, M. J. N. Composição florística da floresta ciliar do rio Mandassaia, Parque Nacional da Chapada Diamantina, Bahia, Brasil. Rodriguésia. v. 60, n. 2, p. 265-276, 2009. Disponível em: < Stable URL: http://www.jstor.org/stable/23499988>.

RIBEIRO, H. F. Caracterização das APP'S dos Córregos Inseridos no Perímetro Urbano da Cidade de Gurupi-TO. In: Seminário de Iniciação Científica da UFT,9. Anais... v. 5, 2013, 2013.

SILVA, W. M. Levantamento das espécies de dois trechos de mata de galeria na sub-bacia do Taquaruçu Grande, município de Palmas- TO: Composição, estrutura e relação planta-solo. Dissertação de Mestrado, Programa de Pósgraduação em Ecologia de Ecótonos da Fundação Universidade Federal de Tocantins, 44p. 2012.

SILVA, W. M., LOLIS, S. F., VIANA, R. H. O. Composition and structure of the gallery forest in the Taquaruçu Grande Sub-basin, Municipality of Palmas, Tocantins State. Acta Scientiarum. Biological Sciences. Maringá, v. 38, n. 1, p. 17-24, 2016. Disponível em: < http://www.uem.br/acta>. DOI: 10.4025/actascibiolsci.v38i1.28663.

SPECIESLINK. Sistema distribuído de Informação que integra em tempo real, dados primários de coleções científicas. 2017. Disponível em: < http://splink.cria.org.br/>. Acesso em Janeiro de 2017.

TARKESH, M.; JETSCHKE, G. Comparison of six correlative models in predictive vegetation mapping on a local scale. Environmental and Ecological Statistics, v. 19, n. 3, p.437-457, 2012. Disponível em: < https://link.springer.com/article/10.1007/s10651-012-0194-3>. DOI: 10.1007/s10651- 
012-0194-3 The International Plant Names Index. 2012. Disponível em: http://www.ipni.org/. Acesso em 26 de Março de 2017.

WERNECK, F.P., NOGUEIRA, C., COLLI, G. R., SITES JR, J. W. \& COSTA, G. C. Climatic stability in the Brazilian Cerrado: implications for biogeographical connections of South American savannas, species richness and conservation in a biodiversity hotspot, Journal of Biogeogrphy, 39, 1695 - 1706. 2012. DOI: 10.1111/j.1365-2699.2012.02715.x. Disponível em: http://onlinelibrary.wiley.com/doi/10.1111/j.1365-2699.2012.02715.x/full>.

WILLIAMS, J. N.; SEO, C. W.; THORNE, J.; NELSON. J. K.; ERWIN, S.; O'BRIEN, J. M.; SCHWARTS, M. W. Using species distribution models to predict new occurrences for rare plants. Diversity and Distributions, v. 15 , n. 4, p. 565-576, 2009. Disponível em: < http://onlinelibrary.wiley.com/doi/10.1111/j.14724642.2009.00567.x/full>. DOI: 10.1111/j.1472-4642.2009.00567.x.

WORLDCLIM - Global Climate Data. Free climate data for ecological modeling and GIS. 2017. Disponível em: < http://www.worldclim.org/>. Acesso em Janeiro de 2017. 